\title{
Simulation tools to assess the impact of Wind turbines on RADAR and other electromagnetic signals
}

\author{
Karen von Hünerbein ${ }^{1}$ Nicolas Douchin ${ }^{2}$, Werner Lange ${ }^{1}$ \\ 1. Lange Electronic GmbH, Rudolf-Diesel-Str. 29A, D-82216 Gernlinden \\ kvh@lange-electronic.com \\ 2. Oktal-Synthetic Environment, 11 Avenue du Lac, \\ 31320 Vigoulet-Auzil, Frankreich \\ nicolas.douchin@oktal-se.fr
}

\begin{abstract}
In recent years the amount of wind energy power plants has risen dramatically, driven by the urgent need to increase the percentage of energy production from renewable, eco-friendly sources. Thus, more and more on-shore and off-shore wind turbine fields have been installed. While this increase is very desirable from an ecological climate perspective there are also sideeffects as noise levels, a threat to birds' lives and impact on the propagation of electromagnetic waves, such as RADAR signals, air traffic navigation signals, and frequencies used for data transmissions, e.g. from UAVs (Unmanned Aerial Vehicles) to the ground and back. Air traffic control RADARs, for example, need to be able to detect and track all aircraft near the airport, especially those flying at low altitude.
\end{abstract}

Detrimental effects include shadowing effects and scattering of signals, as well as increase in EM noise due to scattering, leading to degradation and even loss of the wanted signal, handicapping the data transmission between aircraft and ground stations and detection of flying objects by ground-based RADAR.

In order to be able to assess the wind turbines' impact and degradation of the signals, sophisticated computer simulation tools have been developed [1] which perform physically correct calculations of signal propagation, including geometrical and physical optics [12], with relation to a high resolution 3D environment accurately representing both the terrain including all man-made objects such as the wind turbines and the moving land and air vehicles.

In this presentation we will explain the concept and the background of one such simulation tool and present images and videos of UAV with and without interference caused by wind turbines. We will also show examples of the impact on wind turbines on Signals at 433 and $915 \mathrm{MHz}$, as used by UAVs for telemetry.

\section{Keywords}

Simulation, RADAR signals, telemetry signals, shadowing, interference, security

\section{Introduction}

In order to produce climate friendly electricity, more and more wind energy power plants and wind farms have been built in recent years on-shore and off-shore. German energy produced has increased from 105 TWh (Tera Watt hours) in 2017 to 131.8 TWh in 2019, with 29.456 installed wind turbines on-shore, in Germany alone [2]. Wind turbines represent large structures, with static and moving parts, and varying heights, some of them as much as 160-200 m high. Masts consist of steel, and sometimes of concrete. Hubs and the blades consist of glass fibre reinforced carbon and or metal. 
In Germany, many wind farms, which are fields of multiple wind turbines, are distributed across the country side, which need a minimum distance of 1-15 km to the next airport according to German regulations, a distance, which is different in different states of Germany. No new wind turbines and farms can be built in a radius of $15 \mathrm{~km}$ around a D/VOR navigation station [7]. All new wind turbines and farms need an official permission by the German aviation authority (Luftfahrtbundesamt), before installation, to avoid dangerous Electromagnetic interference and shadowing, as well as mechanical interference with low flying aircraft during take-off and landing. Currently there are around 2.100 wind energy power installations near airports [8].

There is are huge amount of evidence that wind turbines and wind farms can interfere with RADAR signals of ground based RADAR, e.g. for airport surveillance RADARs and air traffic control. Effects on RADAR signals include shadowing and masking, backward clutter, meaning unwanted return signals from wind turbines, distortion and fading of signals. $[3,4,5,6]$. Shadowing means that the wind turbine is large enough to block the return signal from an aircraft. Masking means that the wind turbines act as obstacles. Backward clutter is caused by the reflections from the different parts of the wind turbines and adds enough noise to the return signals to make detection of the wanted target signal difficult or impossible. There are similar effects on a variety of aviation navigation signals (VOR, DME, TACAN), communication signals (VHF, UHF) and airport landing systems, such as ILS and MLS [5].

The effects are more pronounced for low-flying aircraft, e.g. during landing approach or take off, especially near airports impairing the return of the RADAR signals of ground based airport surveillance RADARs [4]. As these effects and unwanted additional signals can endanger normal flight operations near and at the airport, there are regulations in different countries, prescribing minimal distance of wind farms to the airport.

Civil UAVs generally fly at lower altitudes and rely on remote control by ground based pilot (uplink) and transfer data back to user on the ground (downlink) on standard telemetry bands such as 395 $\mathrm{MHz}, 420-450 \mathrm{MHz}$ or $902-928 \mathrm{MHz}$. In these cases, wind turbines can also block or partly block line of sight between the transmitter and the receiver, and add noise and unwanted additional signals to the transmission.

Simulating the effects on the signal propagation of RADAR, telemetry and other EM signals prior to building and installing new wind farm and/or new RADAR and navigation sites, can help to avoid interference and technical problems in the field. The goal is to assess the impact of Wind Turbines on signal propagation of RADAR signals and other RF signals, before installation and operation of new wind turbines and wind farms close to airports, aviation navigation aids and all sorts of RADARs. Another goal is to improve signal detection and processing algorithms both in RADARs and in on-board RF receivers or UAVs and aircraft for telemetry and communication signals, and thus save time and cost in the end.

In order to achieve these goals, a computation tool with graphical output has been developed. It combines the methods of 3D geospecific terrain modelling, simulation of the propagation of electromagnetic signals with raytracing and RADAR modeling $[1,12]$. In this paper we will introduce this tool, and show examples of simulations for signals of a ground based RADAR for UAVs and 
airplanes. We will also show examples of the impact on wind turbines on Signals at 433 and 915 $\mathrm{MHz}$, as used by UAVs for telemetry.

\section{Signal Propagation Modeling Tool}

The computation tool consists of two parts: a signal propagation module and a RADAR modeling module. The RADAR modeling is usually performed by the RADAR manufacturer and is included into the tool as a plugin. The signal propagation module is publicly available and combines the methods of 3D geo-specific terrain modeling, 3D wind turbine and wind farm modeling and the simulation of the propagation of electromagnetic signals with raytracing and physical optics. The tool was developed by Oktal-SE in cooperation with French research institutes and is called SEWorkbench-RF.

In the first step a 3D environment is modeled by importing satellite imagery, digital elevation models and different static and moving objects, such as buildings, trees, UAV or ground vehicles, and the RADAR emitter itself. The 3D model consists of millions of polygons and is made to be as representative of the geographical landscape as possible. It can be very large, e.g. $130 * 130 \mathrm{~km}$, and takes into account the curvature of the Earth resulting in a correct RADAR horizon. Each of the polygons is classified by assigning a physical material to it, with the electromagnetic properties from the SE-WORKBENCH materials database, e.g. reflectivity and back scattering coefficient.

EM signal propagation is calculated in a deterministic way with the following methods:

- geometrical optics

- physical optics

- edge diffraction ECM

- monostatic RCS (Radar Cross Section) computation

- bistatic RCS computation for each reception point

The solution considered here has been developed jointly by OKTAL-SE and ONERA and is dedicated to the simulation of raw data of large-scale environments including complex targets for several kinds of RADAR.

\section{State of the art}

Computations are based on an electromagnetic simulation code based on ray tracing and asymptotic methods [9]. These methods are less physically rigorous than "exact" methods that are strictly based on the resolution of Maxwell equations. However, asymptotic methods enable to handle complex scenes that are very large compared to the wavelength with enough accuracy. In such situations, asymptotic methods provide main contributions of the electromagnetic field which are correctly represented.

In our solution, rays are traced from transmitters towards reception points. These rays are grouped four by four in beams. Rays are traced from transmitters through a grid (figure 1). The intersections of theses beams are computed. There are two types of interactions (figure 2):

- Geometrical Optics (GO), when the beam is reflected by a metallic or dielectric surface

- Physical Optics (PO), when part of the beam energy is scattered towards the reception points at each interaction. 


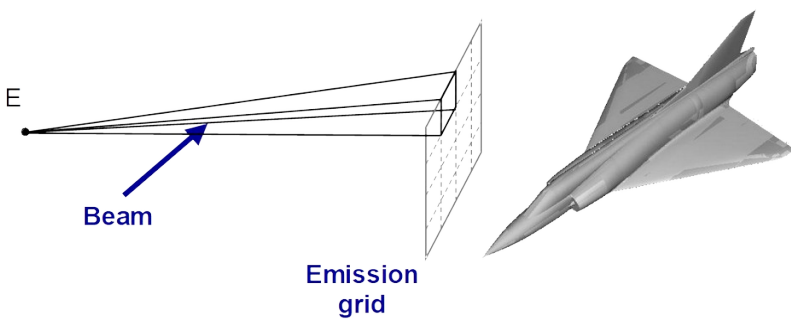

Figure 1. SE-RAY-EM emission grid

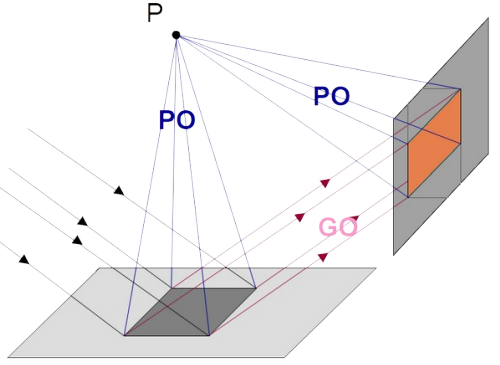

Figure 2. Principle of beam interactions

The ray tracing based on shooting and bouncing ray technique is used to identify the EM contributors in the scene. In the very simple case illustrated by the figure here after (fig. 3) composed by two flat polygons and a unique ray tube, two EM contributors are identified. Then the amplitude and phase of the EM fields scattered to the receiving point by each EM contributor are computed using PO.

Scattering to the receiving point

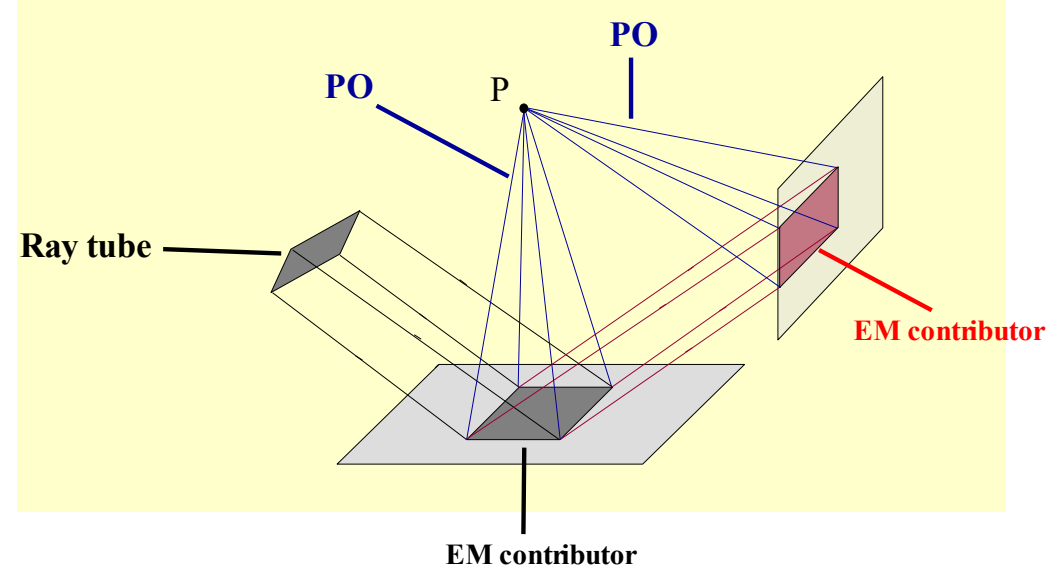

Figure 3. Concept of EM contributor

Validation is achieved through comparison of our solution with other codes based on different methods: typically, an ONERA code based on the Method of Moments to solve the integral equation, called MAXWELL3D [10]. Validation is also based on comparison between simulation and measurements, in cooperation with Fraunhofer FHR for example, as illustrated hereafter (fig. 4):

Validation through comparison

with measured data

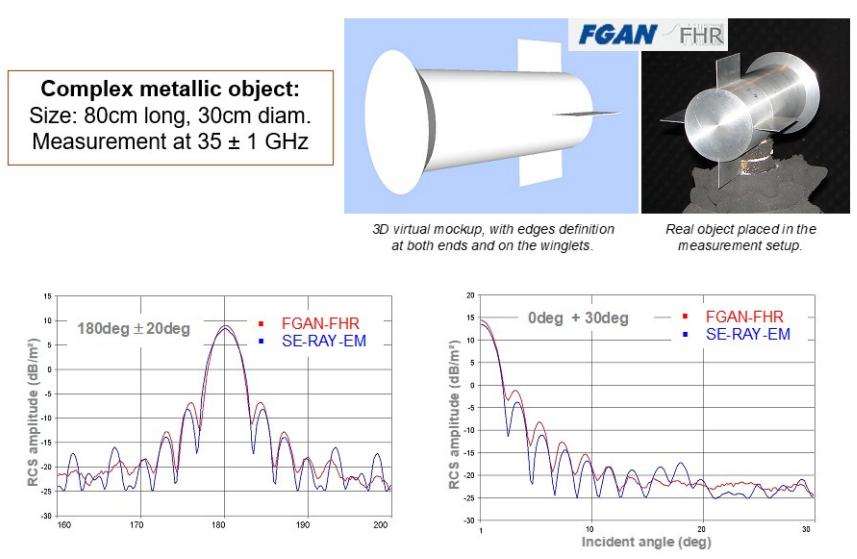

Figure 4. Example of validation test 


\section{Reduction of the computation time through GPGPU implementation}

Nowadays, Graphic Processors Unit (GPU) have proven to be very efficient for optimizing General Purpose (GP) computations, and particularly ray tracing applications [11]. However, the transition to GPU is not straightforward and several issues have to be taken into account.

\section{Double versus Float}

Since GPU are designed to be very efficient for single precision floating point (float) computation, our GPGPU implementation only uses double when it is absolutely necessary, mostly in the computations that involve the phase of the electromagnetic signal.

\section{Cone tracing}

Instead of tracing individual rays, we use cones to trace beams. This way of anti-aliasing is more reliable and the initial resolution can be really coarse. Another advantage of using cones is that they can be processed independently of their neighbors, which is very interesting for parallelization.

\section{Multiple frequency cases}

Multi-frequencies computations consist in computing several frequencies at the same time. Frequencies are independent in terms of electromagnetic computations, but share all the geometric computations. In our GPGPU version, frequency computations are postponed as long as possible. The anti-aliasing process generates all geometrical contributors. These contributors are then used multiple times for applying EM models, one time per frequency.

\section{Validation of the GPU implementation}

The GPU implementation is tested versus the CPU one in terms of both accuracy and performances.

The first test consists in comparing qualitatively the results between the new implementation and both the standard version of SE-RAY-EM and the reference from the MAXWELL3D software of ONERA. The test consists in the computation of the Radar Cross Section (RCS) computation of an aircraft for a frequency of $600 \mathrm{MHz}$ (as represented in figure 5). Figure 6 shows that globally results computed by the three codes are very similar.

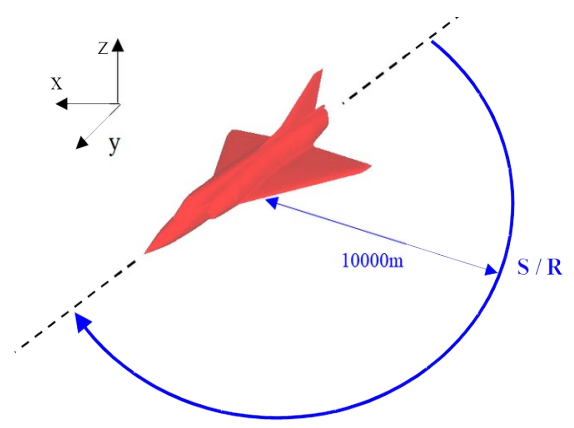

Figure 5. Aicraft RCS computation setup

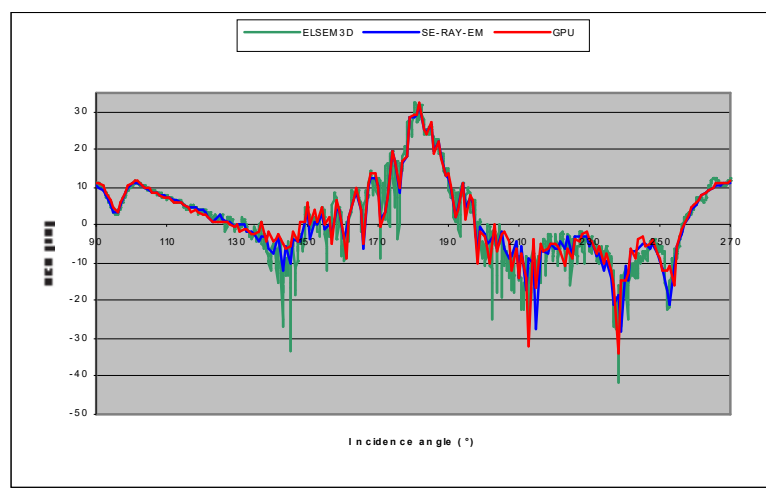

Figure 6. RCS of an aircraft computed with ELSEM3D (green), SE-RAY-EM (blue) and GPU version (red) 
The GPU implementation is 10 to 100 times faster than the standard SE RAY-EM version, depending on the capacities of the graphic board as illustrated in [12].

\section{Physical modelling of the environment}

Any entity of the scene (terrain, wind turbines, mobile entities ...) is considered through a geometrical model (e.g. external surface of a vehicle) and then one or several physical materials are assigned to this geometrical model.

In the simulation presented hereafter, the wind turbines (WTs) are supposed to be entirely metallic which is a worst-case compared to the real life. It means that the physical material assigned to the WTs is a Pefect Electric Conductor (PEC) material.

Concerning the terrain, texture classification is used to efficiently assign physical materials to the 3D model. Textures derived from measured aerial or space photos are mapped on geometries to improve the quality of the appearance of the 3D environment, but also to assign physical materials to the terrain by associating different materials to different colours identified in the image: this is what we call texture classification.

\section{Scenario edition}

Entities of the environment are gathered to compose a virtual scene, shooting conditions are defined, trajectories of mobile entities are created, temporal behaviours and events are handled and the scenario edition tool also enables to interactively visualize the scenario while running.

\section{Scene rendering}

For each sensor defined in the scenario, the physical signal received by the sensor is computed at a given date or over a time interval using realistic rendering (priority put on the precision of the computed signal). The scene rendering consists in computing the EM signal in range gates or as a function of angle of arrival or speed (Doppler shift).

The main advantage of using so complex $3 d$ databases in RADAR simulation is that the real direct interaction of the incident EM wave and the $3 d$ scene can be simply taken into account. This enables one to treat in the same run the target and the background, which is very interesting to naturally deal with the complex interaction of the target with its background.

\section{Special focus on the modelling of WT}

The WT 3D model is decomposed in several parts which are:

- The mast in blue (see the picture hereafter of the WT 3D model, figure 8)

- The fixed part of the hub in yellow

- The rotating part of the hub in green

- The blades in red. 


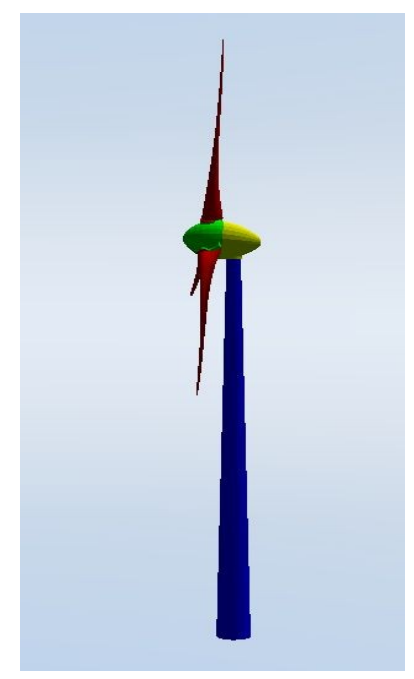

Figure 8. Decomposition of the 3D model of the WT

This decomposition allows one to define rotation for orienting the plane of the blades facing the wind and the make the blades rotating during the simulation. For defining the rotation of the blades Python scripting is used as illustrated on figure 9 hereafter.

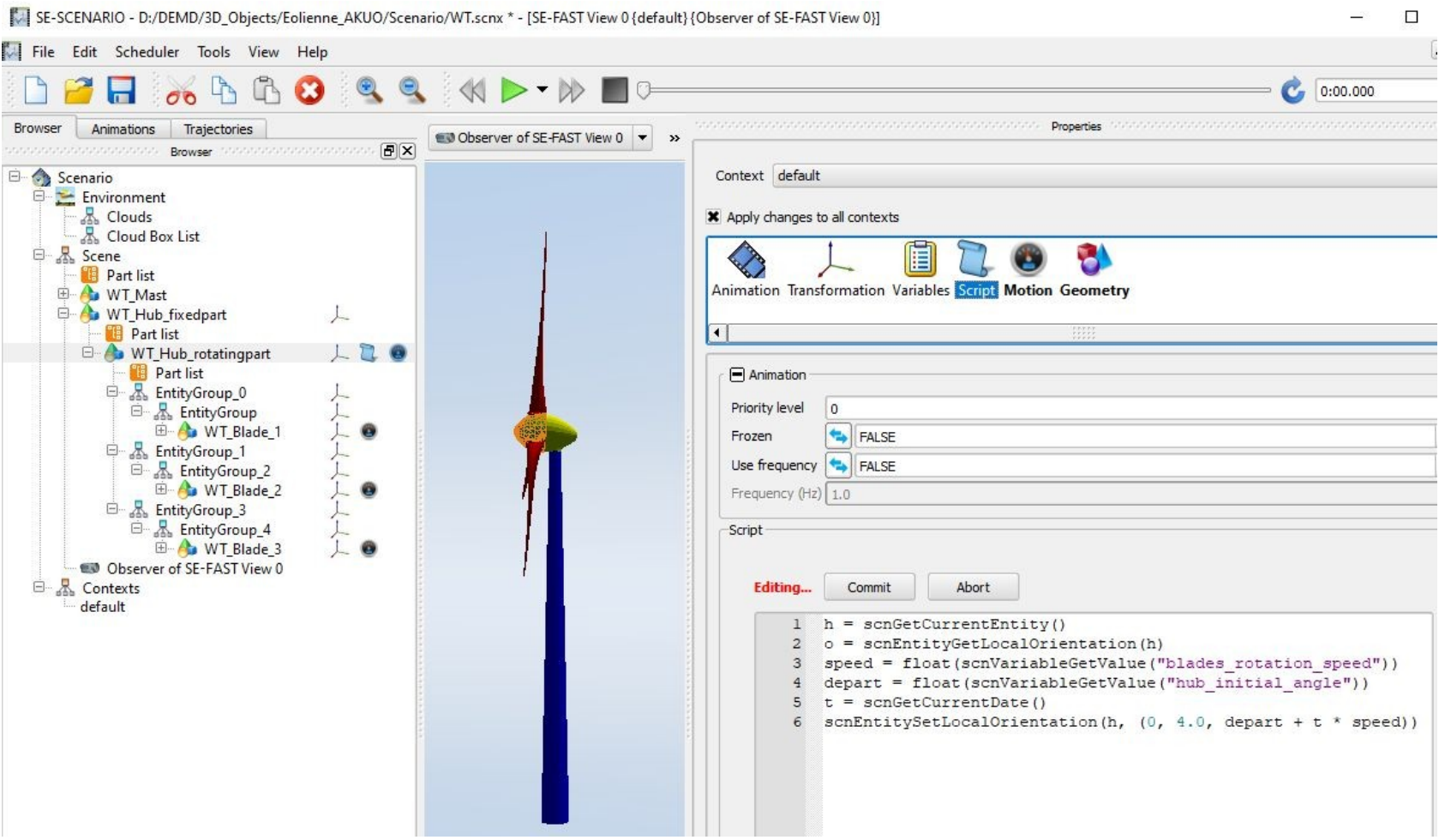

Figure 9. Python script used to define the rotation of the blades 


\section{Wind directions considered in the simulations}

In the simulation results presented hereafter, two wind directions have been considered:

- Wind blowing from the West $\left(W D=-30^{\circ}\right)$

- Wind blowing from the South $\left(W D=-120^{\circ}\right)$.

The orientation of the 12 WTs of the wind farm considered in this work is adapted accordingly as illustrated hereafter (figures 10 and 11):

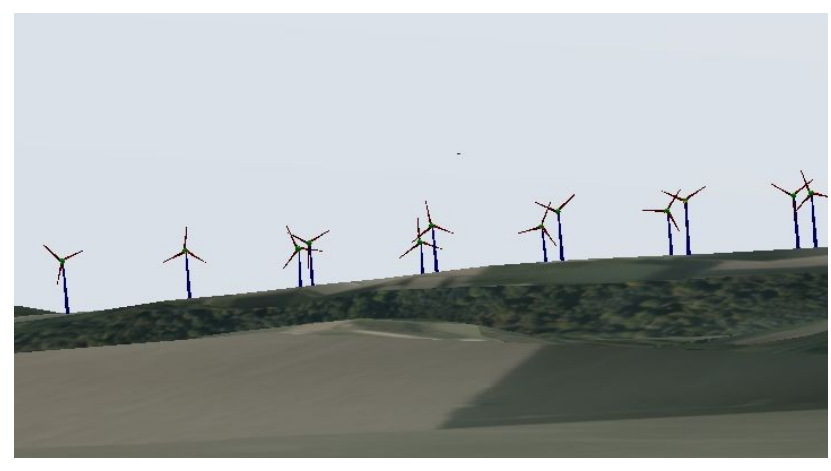

Figure 10. Wind blowing from the West

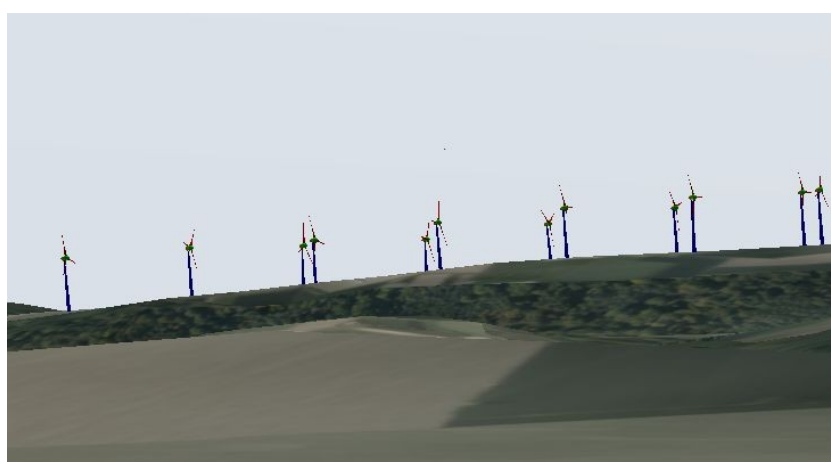

Figure 11. Wind blowing from the South

\section{Scenario description}

The scenario considered in this work is composed by (see figure 12 hereafter):

- the environment (hilly terrain) close to Lyon Saint-Exupery airport

- A wind farm made of 12 WTs put on a hill

- A target which is a drone (quadrocopter) flying along a trajectory at $25 \mathrm{~m} / \mathrm{s}$ speed

- A RADAR (in blue color) installed in the vicinity of the airport, dedicated to air surveillance.
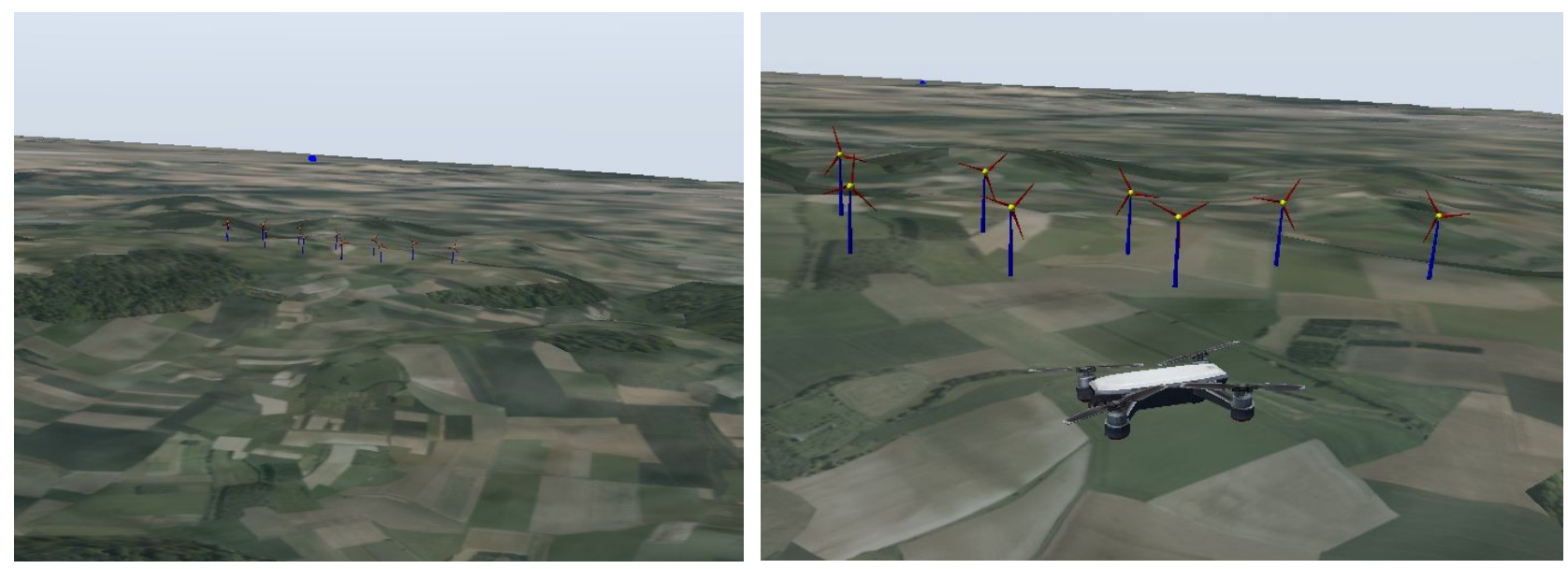

Figure 12. Views of the scenario (the RADAR corresponds to the small blue box in the background) 


\section{Time-Doppler computation in monostatic configuration}

The results presented hereafter (Figures 13 to 16) were derived from the computation of the RADAR echoes generated by the whole dynamic scene considering the Doppler shift due to the rotation of the blades of the WTs and the flying target, as function of time. The time sampling is $100 \mathrm{~Hz}$ and the radar carrier frequency is $5.6 \mathrm{GHz}$.

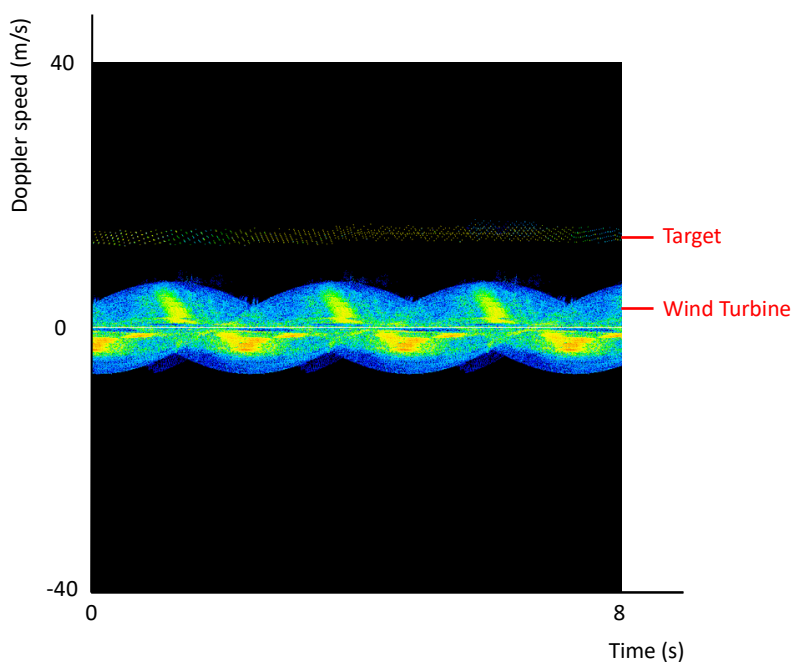

Figure 13. One WT and WD $=-30^{\circ}$ (from West)

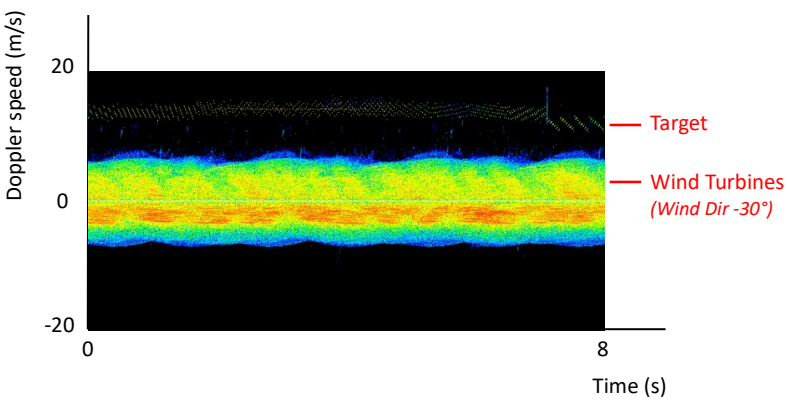

Figure 15. 12 WTs and WD $=-30^{\circ}$ (from West)

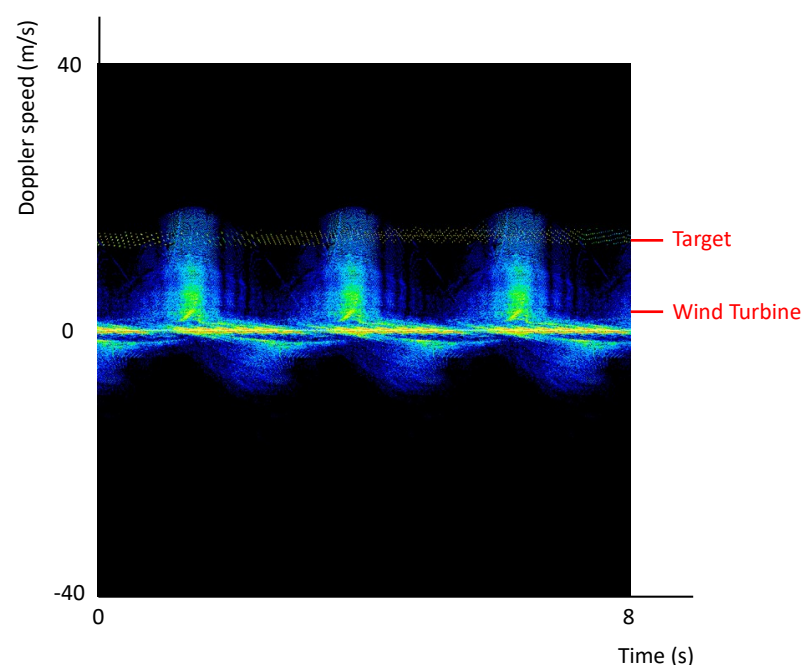

Figure 14. One WT and WD $=-120^{\circ}$ (from South)

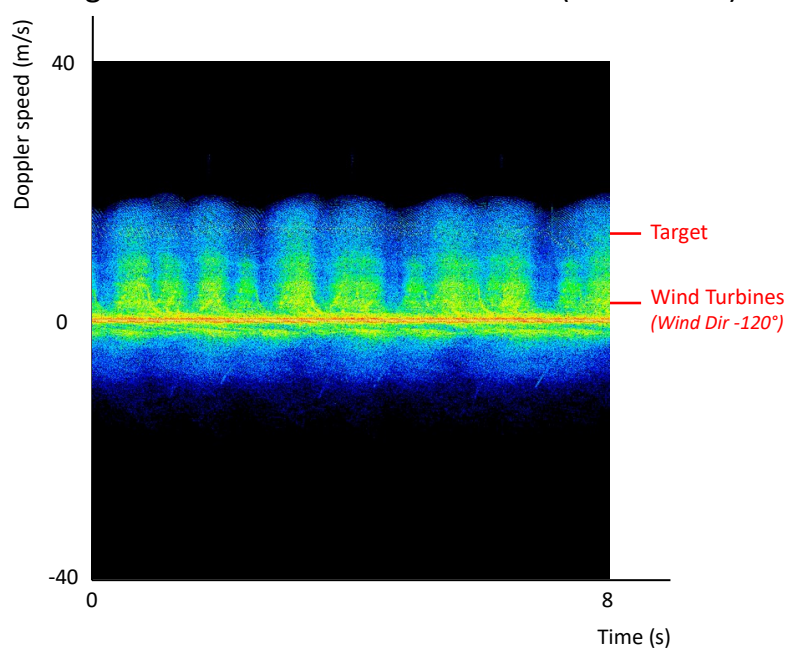

Figure 16. 12 WTs and WD $=-120^{\circ}$ (from South)

It can be observed that the RADAR echoes generated by the WTs is stronger when the blades facing the radar, meaning when wind is blowing from the West. But the Doppler shift due to the rotation of the blades is higher when the blades plane is parallel to the RADAR Line-Of-Sight, meaning when wind blowing from the South. In this situation, it is trickier to detect the target since its Doppler shift is the same range as the Doppler shift of the rotating blades.

It can also be observed that the Doppler signature of the wind farm is much more complex than the one of a single wind turbine. 


\section{Range-Doppler computation in monostatic configuration}

Results presented hereafter (Figures 17 and 18) were computed in the same conditions as before.

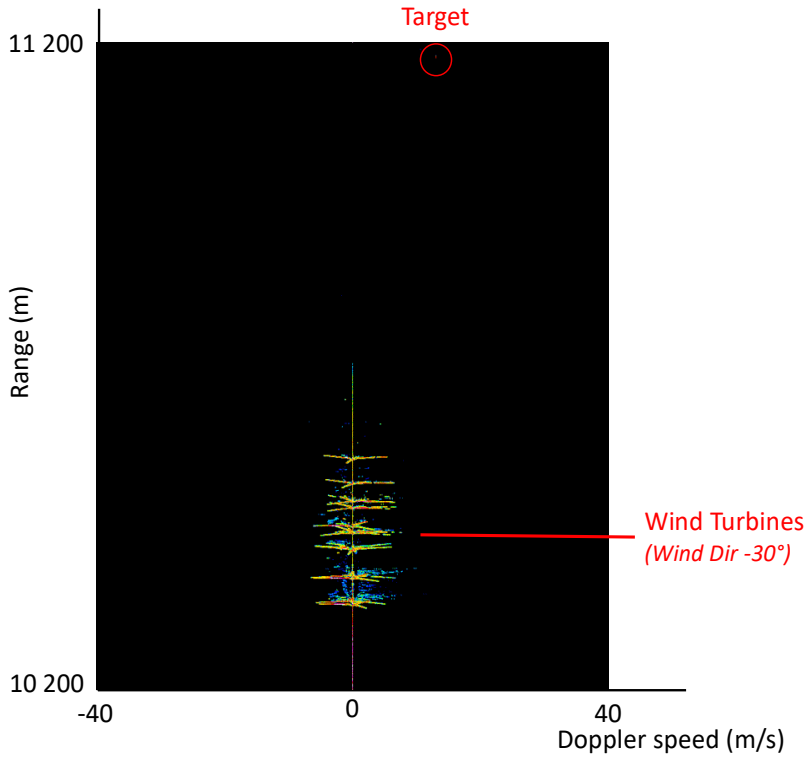

Figure 17. $12 \mathrm{WTs}$ and $\mathrm{WD}=-30^{\circ}$ (from West)

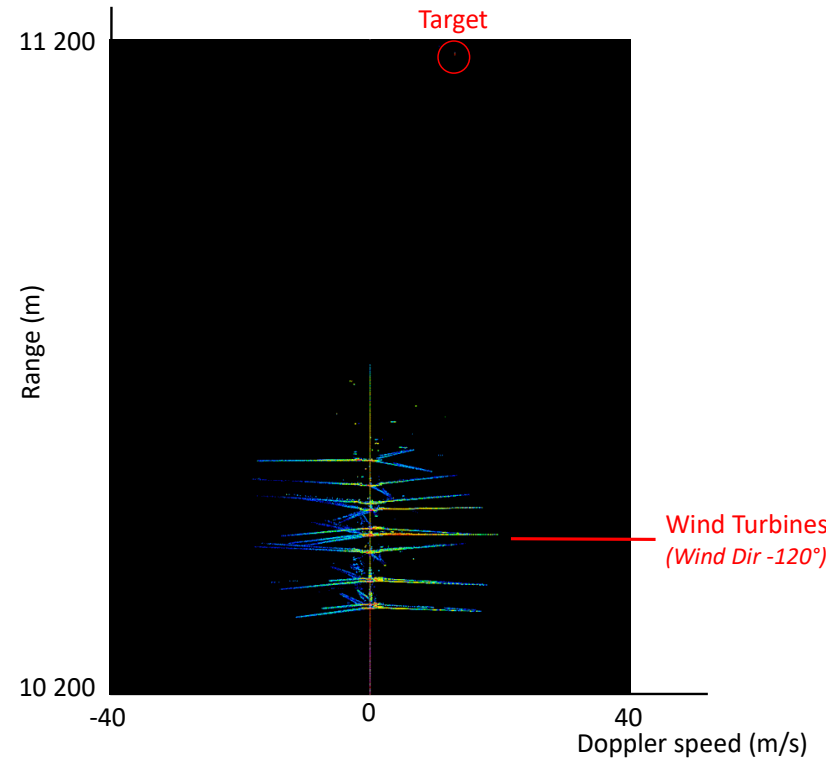

Figure 18. $12 \mathrm{WTs}$ and WD $=-120^{\circ}$ (from West)

Once again, it can be observed that the Doppler shift of the WTs is larger when wind blowing from the South, meaning the blades plane parallel to the radar LOS.

In this Range-Doppler analysis detection of the target is easier in both wind conditions since its range is quite different from the WTs range domain.

\section{Received power computation in bistatic configuration}

The results presented hereafter (Figure 21 to 23) were obtained in bistatic configuration, meaning the receiver is put on the drone target. Two different altitudes were considered for the drone target. At the lowest altitude the drone may be masked by the WTs which is not the case at the highest altitude (see figure 19 and 20 hereafter).

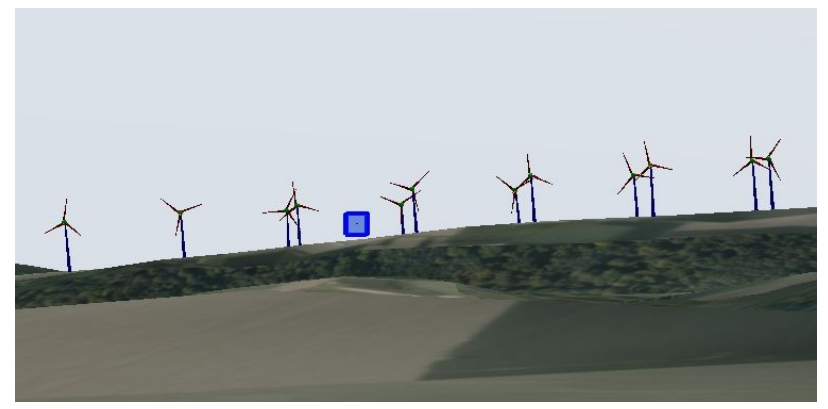

Figure 19. Drone flying at low altitude (100m)

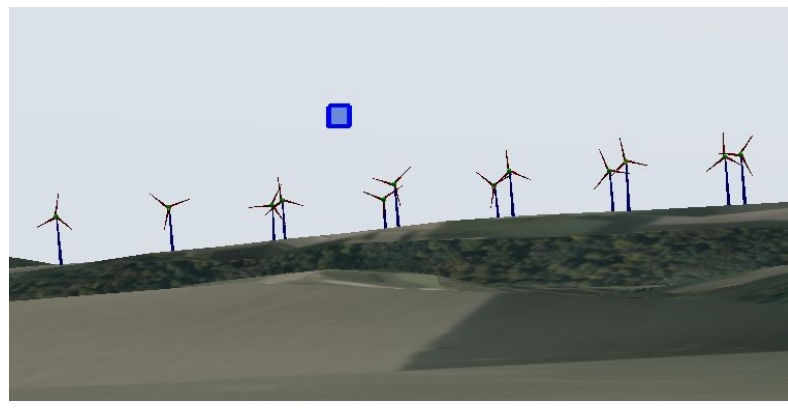

Figure 20. Drone flying at higher altitude (200m)

To obtain the results presented hereafter, the so-called "Forward Scattering" model was used. It is based on the Equivalence Principle which consists in computing the currents induced by the incident wave on all illuminated surfaces. Then we compute the EM fields (scattered fields) radiated by those currents to the receiving point without any occlusion test. The final step is a coherent summation with those scattered EM fields with the fields generated by the wave radiated by the radar directly to the receiving point without any interaction. This approach implemented in our solution allows to compute the EM field in the shadow region of an obstacle. It has been validated with ONERA by comparison with MAXWELL 3D code based on Moment Method. 


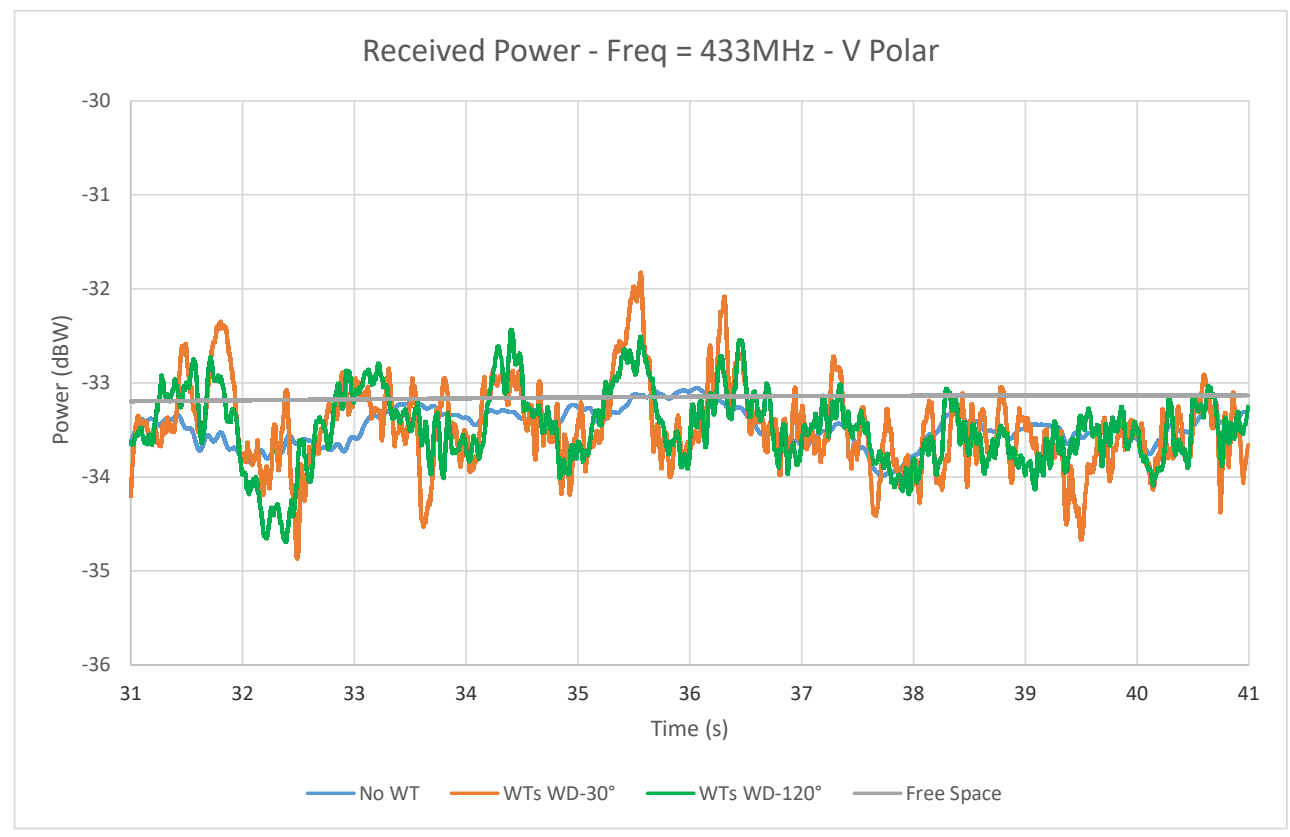

Figure 21. Scintillations at $433 \mathrm{MHz}-$ Drone flying altitude $=200 \mathrm{~m}$

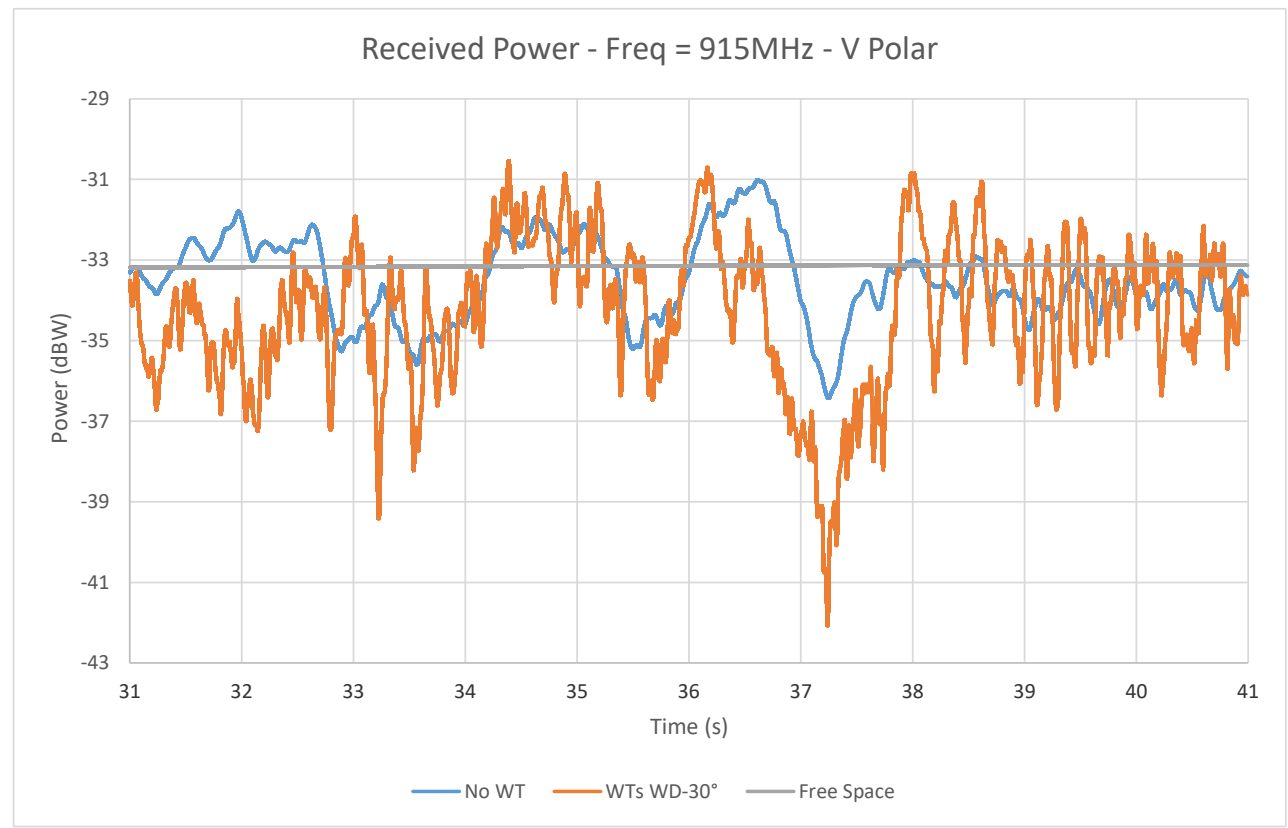

Figure 22. Scintillations and Shadowing at $915 \mathrm{MHz}-$ Drone flying altitude $=100 \mathrm{~m}$

Shadowing can be easily observed at time of the scenario just after $37 \mathrm{~s}$. 


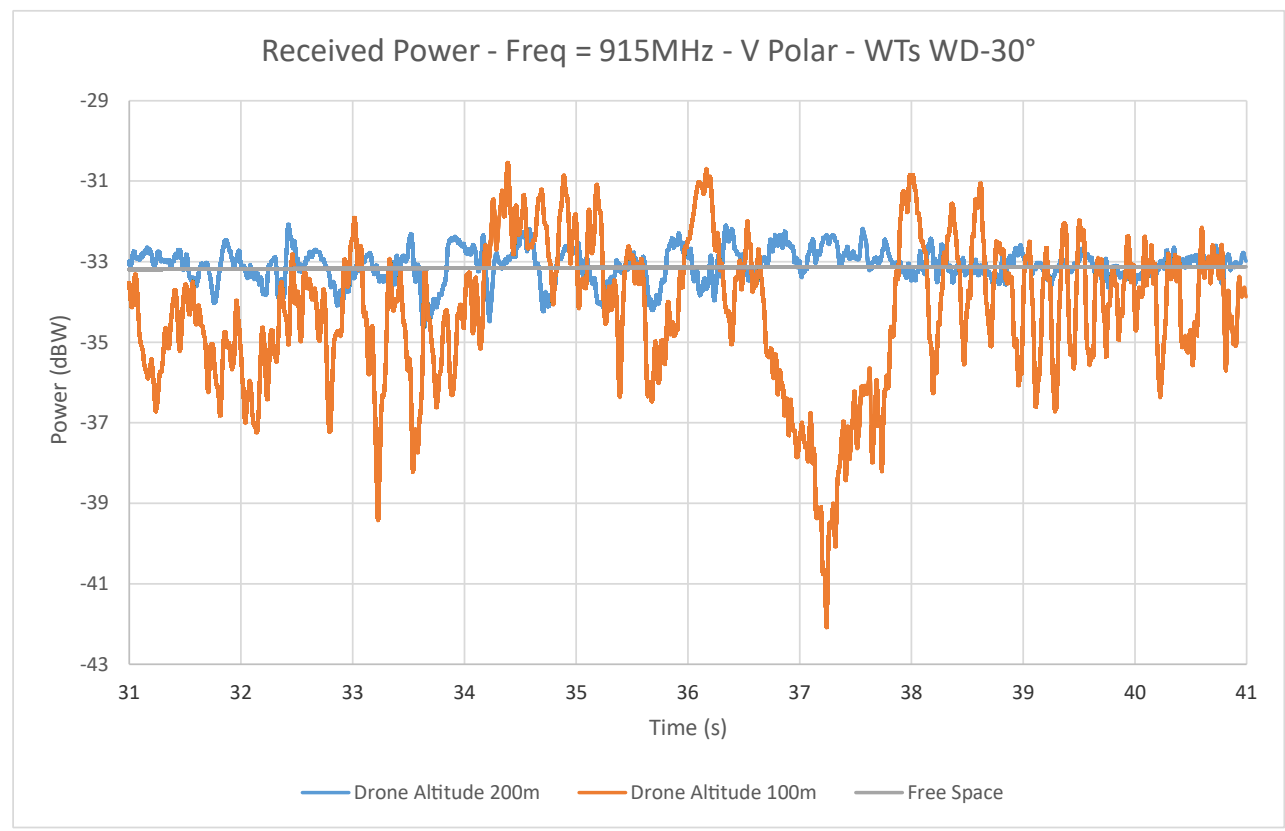

Figure 23. Scintillations and Shadowing at $915 \mathrm{MHz}$

Scintillation is larger when the drone is flying at low altitude. Shadowing observed in low altitude flying situation doesn't occur when the drone is flying at an altitude higher than the wind farm as viewed from the radar (see figure 20).

\section{Conclusions}

High fidelity RADAR simulation is considered more and more as an alternative and complementary methods to real field measurements. Here we have shown a new simulation tool for efficiently simulating the signal propagation of RADAR signals in the vicinity of wind farms with a high computation speed. However, this tool needs to be further validated in different types of environment with different sensor configurations. Some complex simulations require further modeling, e.g. vegetation. The quality of the simulation depends heavily on the high fidelity of the input data, such as accurate and high fidelity synthetic 3D modeling, physical characterization of materials, and determining their EM parameters correctly, and modeling of the atmosphere close to reality.

Validation of the presented solution has been conducted in cooperation with ONERA in the frame of a project sponsored by the DGA (French MoD). Fitting between simulation and measurements is quite good but results are confidential and can't be presented in this paper.

\section{References}

[1] N. Douchin, C. Ruiz (2019) „Performant and High Fidelity Solution for Complex and Large Radar Scene Simulation Including Wind Turbines", EMWT 2019, Toulouse

[2] https://www.wind-energie.de/themen/zahlen-und-fakten/

[3] A. Brown, C. Home, L. Danoon, R. Palama (2018), University of Manchester, Measurement and Modelling of RADAR signatures of large wind turbine using multiple sensors. 2018 IEEE Radar Conference (RadarConf18), DOI: 10.1109/RADAR.2018.8378767. 
[4] J. Bredemeier, T. Schrade, M. Mihalachi, D. Ulm et al (2017) Effects of Wind Turnbines on RADAR Signals. EMWT 2017 Braunschweig 6.-7.12.2017

[5] G. Greving et al., NAVCOM Consult (2016) Wind Turbines and Systems - Simulation Results and Experience 2016, EMWT 2016, Dec 06-07, 2016 at Fraunhofer FHR in Wachtberg

[6] C. A. Jackson, (2007) "Windfarm characteristics and their effect on radar systems," 2007 IET International Conference on Radar Systems, pp. 1-6, 15-18 October, Edinburgh, UK.

[7] https://www.dfs.de/dfs homepage/de/Flugsicherung/Umwelt/Windkraft/FAQs\%20zu \%20Windkraft/

[8]https://www.mdr.de/nachrichten/politik/inland/windkraft-fuenfzighertz-will-ausbau-flughaefenoffshore-100.html

[9] H.J. Mametsa, S. Laybros, T. Volpert, P.F. Combes, P.N. N'Guyen, P. Pitot, (2004) "FERMAT: A high frequency EM scattering code from complex scenes including objects and environment", Progress in Electromagnetic Research Symposium (PIERS 04), Pisa, Italy 28-31 March 2004.

[10] P. Soudais, H. Steve, and F. Dubois, (1999) "Scattering from several test-objects computed by 3-D hybrid IE/PDE methods", Antennas and Propagation, IEEE Transactions on, Vol. 47, No. 4, pp. 646-653, 1999.

[11] Hasselmann, K. and al., (1973) "Measurements of windwave growth and swell decay", Ergänzungsheft zur Deutschen Hydrographischen Zeitschrift, 1973

[12] N. Douchin, C. Ruiz, J. Israel, HJ. Mametsa, (2019) “SE-Workbench-RF: Performant and HighFidelity Raw Data Generation for Various Radar Applications", Proceedings of IRS2019 Conference, 26-28 June 2019, Ulm, Germany 